\title{
Difficulty of transition to adult care in a patient with tuberous sclerosis: a case report
}

Harumi Yoshinaga $^{1}$, Noriko Hayashibara ${ }^{2}$, Michiko Inoue ${ }^{1}$, Fumika Endoh $^{1}$, Kaori Adachi ${ }^{3}$, Eiji Nanba ${ }^{3}$

${ }^{1}$ Department of Child Neurology, National Hospital Organization Minami-Okayama Medical Center

${ }^{2}$ Department of Radiology, National Hospital Organization Minami-Okayama Medical Center

${ }^{3}$ Organization of Research initiative and Promotion, Tottori University

Keywords: Tuberous sclerosis; Transition; Epilepsy; Non-convulsive epileptic status; Levetiracetam; TSC1 gene; Renal failure

Received: November 5, 2020; Accepted: February 27, 2021

\section{Abstract}

We report on a tuberous sclerosis (TSC) patient with intractable epilepsy and chronic renal failure. In spite of having the characteristic features of TSC (cutaneous manifestations, multiple cystic lesions in the kidneys, and intractable epilepsy with a history of brain surgery), he had been overlooked for a long period of time in an inpatient facility for handicapped persons. He was under management by several physicians, including neurologists for adult patients, without a wellorganized transition plan or efficient support from pediatric neurologists. Genetic testing revealed that the patient had a novel causative gene anomaly on the TSC1 gene. Before receiving adequate treatment for epilepsy, he had had daily tonic seizures and focal seizures. After prescription of levetiracetam (LEV) by a child neurologist/epileptologist, his seizures were reduced dramatically. He also had episodes of eating difficulty, which were diagnosed as non-convulsive status epilepticus by EEG monitoring. With the use of LEV infusion and an increase in daily dose of LEV, these episodes also disappeared. TSC is a multisystemic disease and presents various challenges to physicians due to its wide phenotypic variability. Careful transition of a TSC patient from the care of a child neurologist to the care of a general medical practitioner in an adult setting is a critical issue. This case is an important example showing the difficulty of transition to adulthood in a patient with TSC and epilepsy. 


\section{Introduction}

Tuberous sclerosis (TSC) is a multisystemic disease that is often not recognized by general physicians due to its wide phenotypic variability $[1,2]$. Careful transition of a patient from the care of a child neurologist to the care of a general physician is a critical issue [3, 4]. Chronic neurological diseases including TSC pose challenges for the transition from childhood to adulthood care, particularly due to the fact that the manifestations of the disease may change over time. In addition, many adults with chronic neural disease will not become completely independent due to intellectual disabilities or mental health issues.

We report on a TSC patient with intractable epilepsy and chronic renal failure, who had been overlooked for a long period in an inpatient facility for handicapped persons. This case highlights some of the difficulties and solutions related to the transition of patients with chronic neural disease.

\section{Case Presentation}

The patient, a Japanese male, was 50 years of age when his attending physician was changed from a general physician to a child neurologist/epileptologist. Before that time, several general physicians and adult neurologists had been in charge of him since he was admitted to our inpatient facility of handicapped persons.

The patient was bed-ridden, and showed left hand paralysis. His mental state was approximately that of a 10-month-old infant. He could not speak any meaningful words, but recognized his mother, and enjoyed watching
TV. He had cutaneous manifestations characteristic of TSC: white leaflet-like maculae on his abdomen, many café-au-lait spots on his body, and molluscum fibrosum on his face. His kidney function test showed creatinine of $2.54 \mathrm{mg} / \mathrm{dl}$ and urea $48 \mathrm{mg} / \mathrm{dl}$. His red blood cell count was $345 \times 10^{4}$, and hemoglobin was $10.6 \mathrm{~g} / \mathrm{dl}$, showing mild anemia. Other indices including liver function test and blood sugar were normal. Urine analysis revealed transient microhematuria.

The existence of multiple cystic lesions in the kidney was revealed by an abdominal CT that was performed in 2009, when he was diagnosed by a part-time urologist as having polycystic kidney. His cranial CT showed a cavity with marginal calcification in the right frontal lobe (Figure 1). His EEG showed diffuse slow spike-wave bursts during sleep. $\mathrm{He}$ had several types of seizures including daily tonic seizures.

The medical record system at our hospital was digitized in 2009, and only brief summaries of his history prior to 2009 were available. In a summary written in 2009 , the patient's diagnosis is recorded as intractable epilepsy due to post-encephalopathic state, which occurred in infancy.

A detailed medical interview of the patient's mother revealed several important pieces of information. He had been treated for Lennox-Gastaut syndrome (LGS) during infancy by a child neurologist at a university hospital, after which he became gradually retarded and handicapped. At the age of 15 years in 1982, the patient was moved to the inpatient facility for handicapped persons at our hospital, at which time he underwent surgery to remove a slow growing brain tumor at 

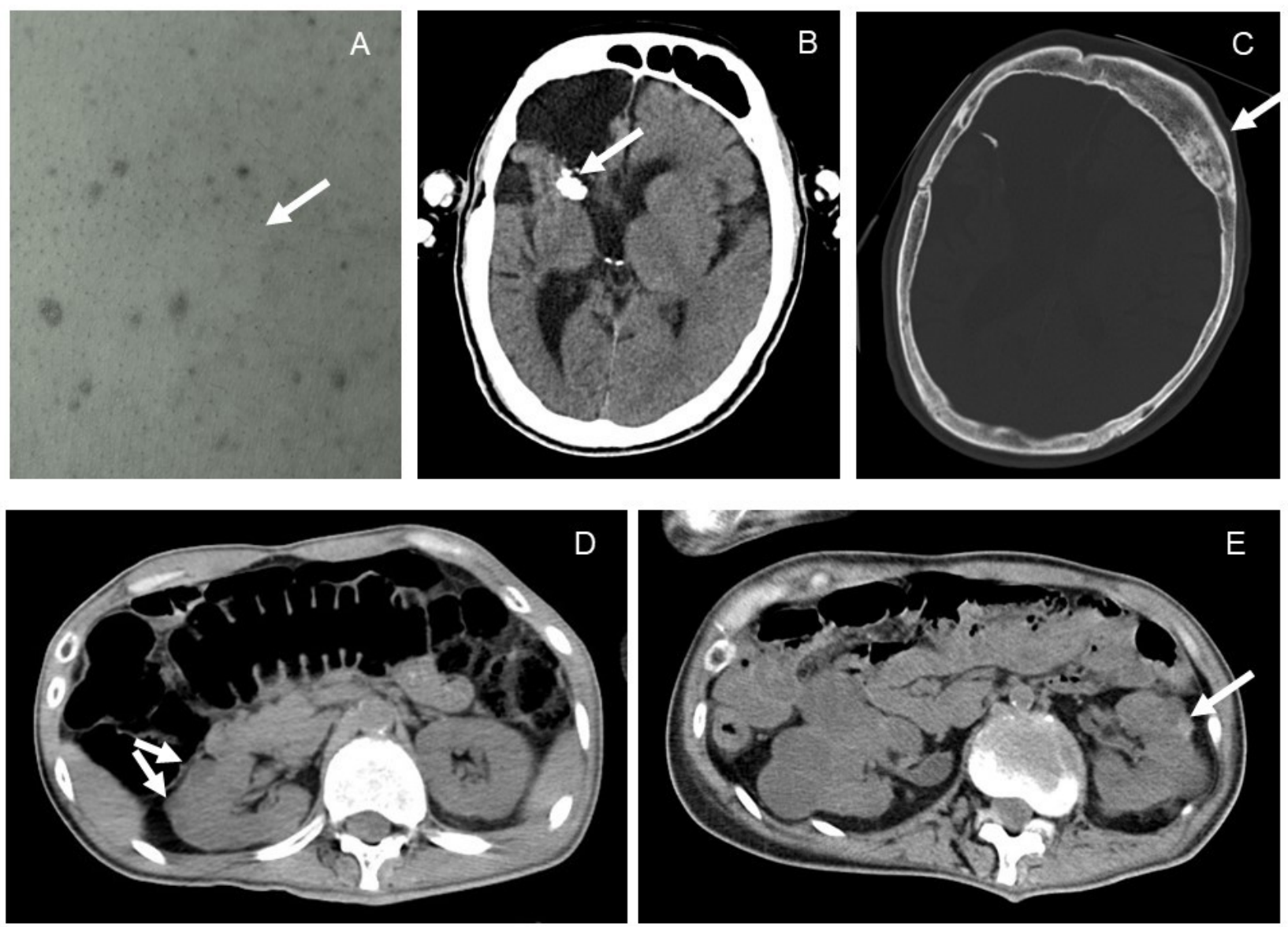

Figure 1. Imaging findings. A. Vague lea flet-like white macula (arrow) and numerous café-au-lait spots on the abdominal skin. B. Brain CT (2017). Right frontal lobe deficit and calcification of the ventricular margin (arrow) are visible. No subependymal nodule (SEN)-like lesions were observed on CT. C. Skull CT (2017) shows sclerotic bone lesions on the skull (arrow). D. Abdominal CT (2009). Bilateral small cystic lesions (arrows) in the left kidney are visible. E. Abdominal CT (2016) shows enlargement of the cystic lesions with bleeding (arrow). No fat component was detected on CT, but this case could be an angiomyolipoma with a limited fat component.

the affiliated university hospital. At that time, his mother was told by the surgeon that the lesion was not a brain tumor, but a lesion with calcified tissue.

With the consent of his family members, the patient's blood was sent to the Tottori University for genetic testing, and a novel variation in the TSC1 gene was found to be the causative mutation, as shown in Figure 2. The genomic DNA was analyzed by a nextgeneration sequencer, MiSeq with TruSight One Sequencing Panel (Illumina, San Diego,
CA, USA). A pathogenic variant of p.Y948Gfs*10 in the TSC1 gene was confirmed by the PCR direct sequencing method. Because of the frame shift by a 5-base deletion, the stop codon is present at a shorter position than normal. This variant is thought to be the cause of the disease.

According to these results, the patient's epilepsy was diagnosed as LGS caused by TSC. He had already been treated with valproate and clobazam. In addition, he was also treated with carbamazepine and phenyto- 


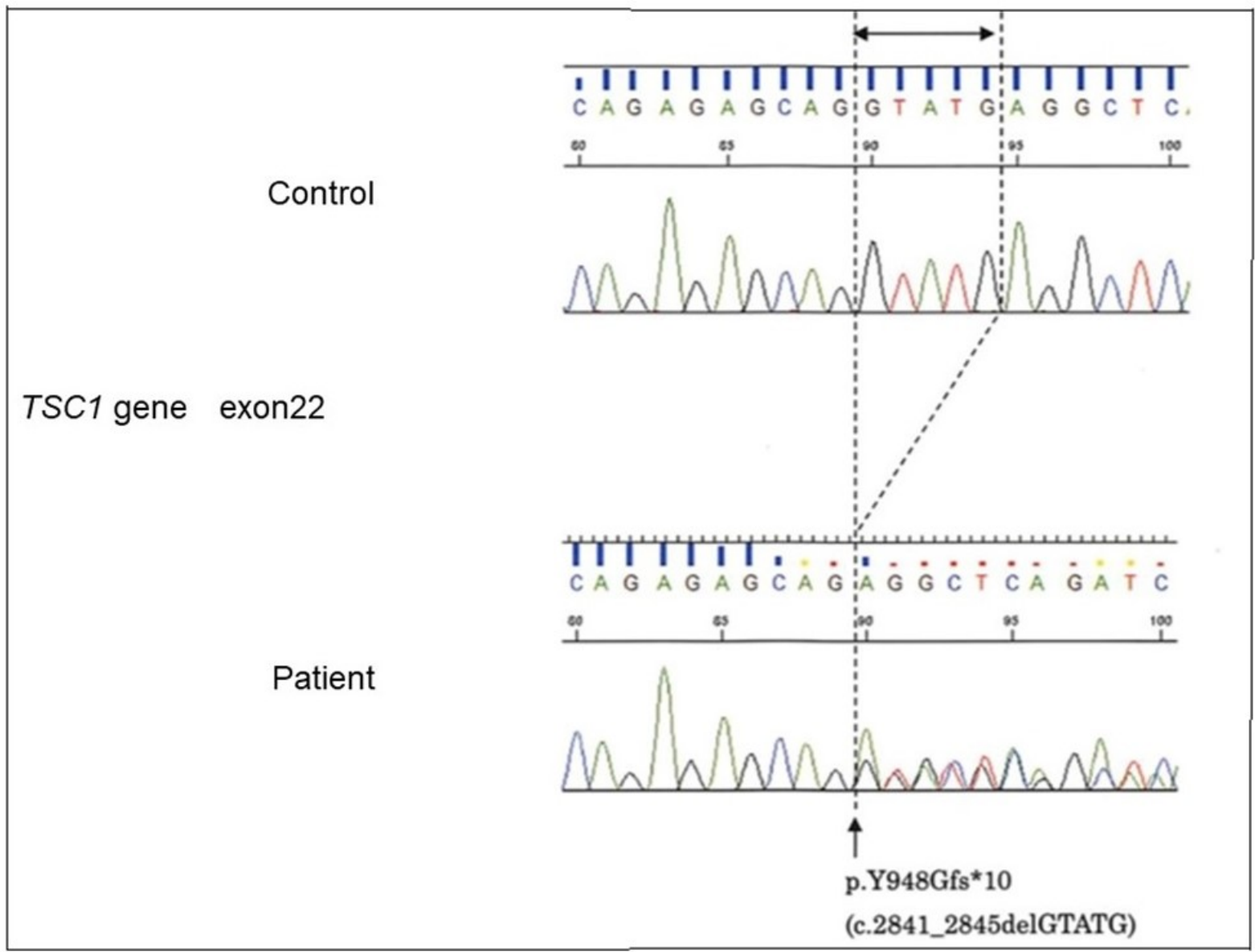

Figure 2. Result of the genomic DNA analysis by a next-generation sequencer, MiSeq with TruSight One Sequencing Panel (Illumina).

in, which were inappropriate for this syndrome. Levetiracetam (LEV) was prescribed instead of carbamazepine because the patient had daily tonic seizures and focal seizures. As a result, his seizures dramatically decreased from several seizures per day to several seizures per month.. Also, he had episodic eating difficulties with impairment of consciousness once every few months, which was diagnosed as non-convulsive status epilepticus (NCSE) by ictal EEG. This was not a form of eating epilepsy, but episodic impairment of consciousness lasting for around one hour. As shown in Figure 3, diazepam infusion had an effect on his EEG during bouts of NCSE. At another time, LEV infusion ameliorated his impairment of consciousness and reduced his confusion, and he started eating his dinner just after the start of the LEV drip infusion. Therefore, his daily dose of LEV was increased, which eliminated these episodes completely.

As for the cystic lesions in his kidneys, we believed them to be angiomyolipoma with TSC, and proposed treatment with everolimus, which is the standard therapy for renal lesions in TSC, but his mother refused any further treatment for his kidney lesions, including dialysis, after progression of his kidney lesions [5]. The patient died two years 
after the diagnosis of TSC, because of massive bleeding in his renal cysts. However, his mother was satisfied that he had not suffered from daily epileptic seizures and episodic eating difficulty during the last two years of his life due to treatment with LEV.

\section{Discussion}

The present case highlights the problems encountered in the transition from childhood care to adult care for patients with chronic disease. Advances in medicine have also allowed people suffering from chronic childhood diseases, such as diabetes, asthma, and malignant diseases, as well as neurological diseases, to survive into adulthood [6, 7]. In this case, the difficulties in transition were caused by several problems. First, the patient's records were not transferred from his pediatric physicians to his adult physicians. Next, the records of the surgery to remove a TSC-related brain lesion were not transferred from the surgeon to his adult-care facility. Moreover, the patient's pediatric physicians noticed the existence of multiple cystic kidney lesions, but performed no further investigations to ascertain their cause. Finally, some of his medical records were lost because the obligation for storing medical records in Japan is only five years.

To address these issues, a personal health record (PHR) system like those used in the USA and Europe to store patient information in a lifelong database that doctors can access

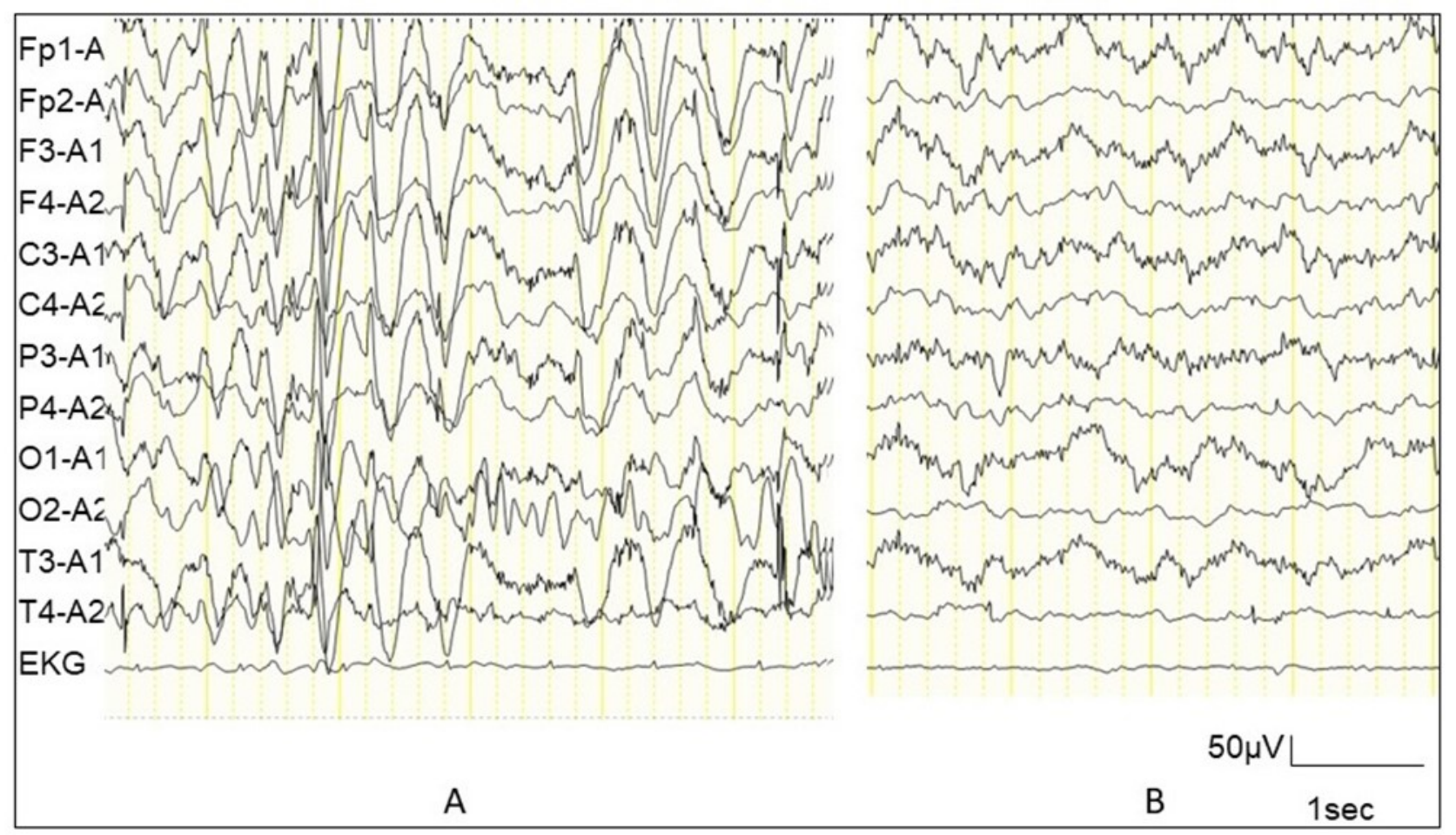

Figure 3. EEG findings. A. EEG during an episode of eating difficulty. Diffuse 2-3 Hz high voltage slow waves mixed with spike-waves appeared continuously, which was different from his usual awaking record. He could not chew or swallow food due to impaired consciousness during these episodes. B. After diazepam infusion, the epileptic discharges and high-voltage slow waves disappeared and the clinical symptoms were dramatically improved. 
at any time is needed [8]. A PHR is most useful for patients with conditions such as diabetes and asthma, who have no intellectual disabilities. Such patients can be trained to take over their own care as they enter adulthood, care which had previously been handled by their parents or other caregivers. To gain such independence, support from schools and health care workers also plays a role in the transition [7]. In contrast, in patients with intellectual disabilities, such as those with TSC, the situation is much more complex, as these patients require lifelong care [9]. Indeed, TSC is a multiorgan disease and patients often face significant barriers in transitioning from a multidisciplinary pediatric health care system to an individually-based adult setting [3, 4]. In order to properly handle the transition of a patient with TSC, knowledge of the natural history of TSC-related manifestations is required for both child and adult neurologists, as well as nephrologists, dermatologists and even general physicians. In this case, the child neurologist was only interested in his intractable epilepsy, while the brain surgeon was only concerned about the brain tumor, and the urologist only noticed the polycystic lesion in the kidney. Therefore, the problems encountered with this patient might have resulted from a failure to make a proper diagnosis due to the lack of communication between the various specialists who treated him. A multidisciplinary clinic or TSC center, as envisioned by Nabbout et al. [9], would be ideal for managing an efficient transition. However, even if a multidisciplinary clinic or TSC center is not available, if physicians start discussing the process of transition early and educating the caregivers about the disease and treatment, the transition is more likely to go well, as noted by Peron et al. [4].

A medical team led by the attending child neurologist should manage the clinicians and caregivers involved in the transition of TSC patients, and should consider the possible medical issues of TSC in adulthood. And, of course, a referral letter to the adult physicians should always be prepared as a starting point [4].

Transition for children with epilepsy to adult care is also challenging because of the variety of epilepsies and multiple comorbidities $[10,11]$, as seen in this case. The etiology of epileptic seizures is known to be an important factor in choosing an antiepileptic drug [12]. However, the etiology of conditions like TSC are not familiar to a general physician. In TSC, other than vigabatrin, which is the most effective drug in the treatment of infantile spasms [13], there is Class IV evidence that everolimus improves seizure control in medically refractory epilepsy [14]. In the present patient, we proposed treatment with everolimus after progression of the patient's renal lesions, but his mother refused. If he had been diagnosed as having TSC and treated with everolimus for his renal lesions before the development of angiomyolipoma, his clinical course might have changed. After his death, everolimus had been approved in 2019 for every symptom including epilepsy related to TSC. Had it been available for use, it might have improved his seizure control. Instead, we tried LEV and it resolved every type of seizures, including non-convulsive status epilepticus. There has been also a report on the efficacy of LEV, which affects the SVR2 protein [15]. And the possible contri- 
bution of the SVR2 protein to the epileptogenicity of TSC has also been reported [16]. Knowing the etiology will lead to suitable treatment for epilepsy.

In addition, the present patient had been treated with various drugs, some of which were unsuitable for LGS. He also had episodic eating difficulty, which had not been diagnosed as NCSE until it was detected by video -EEG recording. NCSE is sometimes observed in LGS, and is well known by child neurologists.

Some forms of childhood onset epilepsy, including LGS, are characterized by seizures that are not usually seen in the adult neurology field. Even in a tertiary epilepsy center, some neurologists may not feel that they are prepared to diagnose and treat adult patients with some childhood onset epilepsies [10, 11]. Moreover, the doctors who work at long -term care facilities are predominantly general physicians. Child epilepsy specialists must organize a plan to evaluate diagnosis, update treatment to resolve polypharmacy, and screen for etiology and comorbidities, before transfer of pediatric patients to adult facilities.

This case is an important example that shows the difficulty of transition to adulthood in patients with TSC and epilepsy.

\section{Conclusions}

Specialists in child neurology or the members of a TSC center should perform various histopathological and radiological investigations to detect multi-organ complications in TSC patients in order to assure a careful transition from pediatric to adult care. Physicians should put in place a suitable treatment plan for patients, including a treatment plan for each organ system, especially in cases of intractable epilepsy. These should be completed before such patients are admitted to longterm care facilities as adults.

\section{Ethical Approval}

Written informed maternal consent was obtained to publish the patient's data in a scientific journal. This study was approved by the Ethic Board at Minami-Okayama Medical Center and the genetic testing was also approved by the Ethical Committee at Faculty of Medicine, Tottori University.

\section{Conflict of Interest Disclosure}

The authors declare no competing interests

\section{References}

[1] Northrup H, Krueger D. The International Tuberous Sclerosis Complex Consensus Group. Tuberous Sclerosis Complex Diagnostic Criteria Update: Recommendations of the 2012 International Tuberous Sclerosis Complex Consensus Conference. Pediatr Neurol 2013;49:243-254.

[2] Krueger D, Northrup H. The International Tuberous Sclerosis Complex Consensus Group. Tuberous Sclerosis Complex Surveillance and Management: Recommendations of the 2012 International Tuberous Sclerosis Complex Consensus Conference. Pediatr Neurol 2013;49:255-265.

[3] Thiele EA, Granata T, Matricardi S, Chugani HT. Transition into adulthood: tuberous sclerosis complex, SturgeWeber syndrome, and Rasmussen en- 
cephalitis Epilepsia 2014;55:Suppl 3:29 -33 .

[4] Peron A, Canevini MP, Ghelma F, Di Marco F, Vignoll A. Healthcare transition from childhood to adulthood in Tuberous Sclerosis Complex. Am J Med Genet 2018;178(3):355-364.

[5] Bissler JJ, Kingswood JC, Radzikowska E, Zonnenberg BA Frost M, Belousova E, et al. Everolimus for renal angiomyolipoma in patients with tuberous sclerosis complex or sporadic lymphangioleiomyomatosis: extension of a randomized controlled trial. Nephrol Dial Transplan 2016;31(1):111-119.

[6] American academy of pediatrics, American academy of family physicians and American college of physiciansAmerican society of internal medicine. A consensus statement on health care transitions for young adults with special health care needs. Pediatrics 2002;110;1304-1306.

[7] Ramdzan SN, Khoo EM, Leiw SM, Cunningham S, Kendall M, Sukri N, et al. How young children learn independent asthma self-management: a qualitative study in Malaysia. Arch Dis Child 2020;105:819-824.

[8] Roehrs A, da Costa CA, Righi RD, de Oliveira KS. Personal health records: A systematic literature review. $\mathrm{J}$ med internet res.2017;19(12):e401-419.

[9] Nabbout R, Belousova E, Benedik MP, Carter T, Cottin V, Curatolo P, et al. Epilepsy in tuberous sclerosis complex: findings from the TOSCA study Epilepsia open 2018;21:4(1):73-84.
[10] Andrade DM, Bassett AS, Bercovici E, Borlot F, Bui E, Camfield P, et al. Epilepsy: Transition from pediatric to adult care. Recommendations of the Ontario epilepsy implementation task force. Epilepsia 2017;58(9):1502-1517

[11] Borlot F, Tellez-Zenteno JF, Allen A, Ali A, Snead OC 3rd, Andrade DM Epilepsy transition: challenges of caring for adults with childhood-onset seizures. Epilepsia 2014;55(10):16591566.

[12] Scheffer IE, Berkovic S, Capovilla G, Connoly MB, French J, Guilhoto L, et al. ILAE classification of the epilepsies: Position paper of the ILAE commission for classification and terminology. Epilepsia 2017;58(4):512-521.

[13] Overwater IE, Bindels-de Heus K, Rietman AB, Ten Hoopen LW, Vergouwe Y, Moll HA, et al. Epilepsy in children with tuberous sclerosis complex: Chance of remission and response to antiepileptic drugs. Epilepsia 2015;56 (8):1239-1345.

[14] Krueger D, Wilfong A, Mays M, Talley C, Agricola K, Tudor C, Capal J, Holland-Bouley K. Long-term treatment of epilepsy with everolimus in tuberous sclerosis. Neurology 2016;87:1-8.

[15] Collins JJ, Tudor C, Leonard JM, Chuck G, Franz DN. Levetiracetam as adjunctive antiepileptic therapy for patients with tuberous sclerosis complex: a retrospective open-label trial. J Child Neurol 2006;21(1):53-57.

[16] Toering ST, Boer K, de Groot M, Troost D, Heimans JJ, Spiliet WGM, et al. Expression patterns of synaptic vesi- 
cle protein $2 \mathrm{~A}$ in focal cortical dysplasia and TSC-cortical tubers. Epilepsia 2009;50(6):1409-1418. 